\title{
Chemical biology for discovery: perspectives and challenges
}

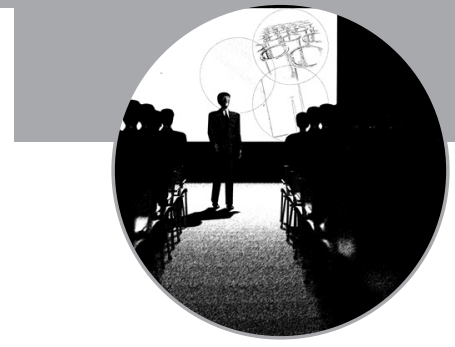

\author{
14th ISCB International Conference (ISCBC-2010) \\ CDRI, Lucknow, India, I5-18 January 2010
}

The Indian Society of Chemists and Biologists, organized its I4th International Conference on 'Chemical Biology for Discovery; Perspectives and Challenges' at the Central Drug Research Institute (CDRI). The four days of scientific programs included nine plenary lectures, 50 invited lectures by eminent scientists and 16 oral presentations. A total of 255 posters were presented by young scientists and $\mathrm{PhD}$ students in three poster sessions. Several scientists presented their work on drug research, chemical sciences, bionanotechology, chemical biology, glycobiology and biochemistry.

Approximately 550 delegates from India and other countries, including the USA, UK, Russia, France, Finland, Switzerland, Germany, Belgium, Sweden, Taiwan and Kuwait, participated in the conference. Most of the speakers presented their work, which included highlights on synthesis, structureactivity relationships (SARs) and current trends in medicinal chemistry and drug research. A brief selection of the highlights is discussed here.

\section{Olefin metathesis reaction}

Robert H Grubbs, Nobel Laureate (California Institute of Technology, Pasadena, USA) delivered a special lecture on the olefin metathesis reaction. He presented the utility of this reaction and discussed the development of new catalysts that are extremely tolerant of organic functional groups. Due to their high activity, functional group tolerance and ease of use, these ruthenium-based catalysts have found wide application in organic and polymer synthesis

\section{Antitrypanosomal activity of nitrogen heterocycles}

Colin J Suckling (University of Strathclyde, Glasgow, UK) presented a talk entitled 'Heterocyclic chemistry at the edge of biology and medicine.' He discussed the chemistry of fused pyrimidines, which include pteridines, pyridopyrimidines and pyrrolopyrimidines, and the chemistry of DNA minor groove binders. The lecture described new diversity-oriented synthetic methods for fused pyrimidines followed by their biological activity, in particular the proof-of-concept for the treatment of trypanosomiasis.

\section{NAD+-requiring enzymes as targets}

Michael D Threadgill (University of Bath, UK) discussed nicotinamide adenine dinucleotide (NAD), one of the most important small-molecule components of the cell. Many important pathways use $\mathrm{NAD}^{+}$(or its reduced form NADH), which is a substrate or co-factor for more than 100 enzymes. He described how several NAD ${ }^{+}$ requiring enzymes have been described and exploited as drug targets. He also discussed the therapeutic opportunities for selective inhibition of $\mathrm{NAD}^{+}$-requiring enzymes. He presented recent results on the effects of inhibition of PARP-1 on cancer metastasis, selective inhibition of the PARP-2 isoform and inhibition of sirtuins.

\section{Challenges \& practical perspectives of peptidomic research}

Vadim $T$ Ivanov (Institute of Bioorganic Chemistry, Russia) delivered a lecture entitled 'Challenges and Practical Perspectives of Peptidomic Research.' He highlighted that the total screening of biological samples for peptides holds considerable promise for elucidating biochemical regulatory networks and for the discovery of new bioactive molecules or disease markers.

\section{Challenges for medicinal chemists}

Leena Otsomaa (Orion Pharma, Espoo, Finland) discussed the productivity of R\&D in the pharmaceutical industry and the decreasing number of approved NCEs as R\&D spending has been continuously growing. She presented that natural emphasis on productivity has put pressure on the key players in drug discovery, including medicinal chemists. The characteristics of medicinal chemistry have changed, along with the possibilities that new technologies have provided for drug discovery. Some characteristics have remained the same, but today's continual increase in urgency has forced chemists to look for new solutions as well as to change their way of working.

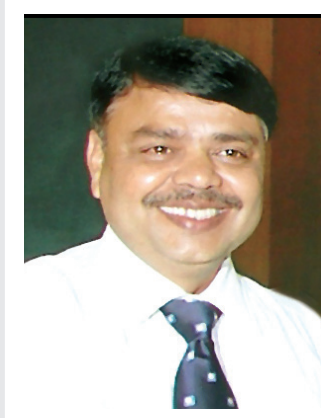

PMS Chauhan General Secretary, Indian Society of Chemists and Biologists (ISCB ), Medicinal and Process Chemistry Division, Central Drug Research Institute, Lucknow 22600I, India

Tel.: +9l 5222439492

Fax: +9l 5222623405

E-mail: premsc58@

hotmail.com;

secretary@iscbindia.org 


\section{Target-to-drug discovery for} novel leishmaniacidals

Neeloo Singh (CDRI, Lucknow, India) spoke of the fact that leishmaniasis is endemic in at least 85 developing countries with at least 1.5 million estimated cases occurring each year and an additional 350 million people at risk from infection. She described the significance of pteridine reductase 1 enzyme as a molecular target in parasite growth and survival. Singh discussed targeting this pathway and described development of a multi-tiered compound screening paradigm to identify and confirm novel leishmaniacidal lead molecules. Transgenic Leishmania were used in a flow cytometry screen, aided by structural modeling of the target recombinant enzyme. She also discussed development of biochemical and biological screening assay formats to identify and characterize new potent Leishmania donovani growth inhibitors targeting the amastigote found in the human host. She described the identification of lead molecules that display in vivo activity without toxicity to human cells. Monastrol, which is an established anticancer drug, was identified as a novel leishmaniacidal lead molecule and confirmed that the leishmaniacidal effect of these molecules is triggered by programmed cell death. The whole genome sequencing of $L$. donovani clinical isolates is currently being carried out in her laboratory.

\section{Role of molecular activation in the generation of new indole reactivity} David St C Black (University of New South Wales, Sydney, Australia) presented on the role of molecular activation in the generation of new indole reactivity. He highlighted that activated 4,6-dimethoxyindoles are capable of being nucleophilic in a variety of positions, including C3, C2 and C7, depending on the overall substitution pattern. The special chemistry of activated indoles allows the formation of diindolylmethanes, triindolyldimethanes and tetraindolyltrimethanes, and these can further lead to interesting macrocyclic structures.

\section{Natural product-inspired probes of DNA \& RNA microenvironments}

Graham B Jones (Northeastern University, Boston, USA) discussed that nucleic acids can have richly diverse structures, including hairpins, knots, pseudoknots, triple helices, loops, helical junctions and bulges. Such bulged structures in nucleic acids are of general biological significance and have also been suggested as binding motifs for regulatory proteins involved with viral replication, including the TAR region of HIV-1. Additionally, the etiology of at least 12 human neurodegenerative genetic diseases has been attributed to genetic variations in the lengths of triplet repeats in genomic DNA (e.g., myotonic dystrophy, Huntington's disease, Friederich's ataxia and fragile $\mathrm{X}$ syndrome).

\section{An enzymatically active fibrillar film of bacteriophage-associated hyaluronate lyase}

Vinod Bhakuni (CDRI, Lucknow, India) discussed that the in vitro assembly of a soluble protein into its mature fibrillar form is usually accompanied by loss of its functional activity. He demonstrated that a natural enzyme (HylP2) retains its enzymatic activity on conversion from prefibril to a mature fibril and supports the contention that minor conformational changes in the native folded form of a protein can lead to the formation of a functional fibril. He discussed that fibrillar film formation owes novelty to hyaluronidase enzymes and its functionality in the organism establishes fibrils as a genuinely acquired protein fold/structure.

\section{Design, synthesis \& small-library generation of benzopyran-related heterocycles \& their in vitro anticancer evaluation}

Anamik Shah (Saurashtra University, Rajkot, India) spoke about the synthesis of coumarins and other pyran analogs and their in vitro anticancer activity. He reported that structural changes in several benzopyran derivatives at the benzenoid moiety in addition to several synthetic modifications at $\mathrm{C}_{3}$ and $\mathrm{C}_{4}$ positions have led to the generation of several new small libraries. The primary screening of these libraries was carried out and screening of selected molecules were done on a panel of cell lines representing various cancers.

\section{Value creation \& new opportunities in medicinal chemistry}

Mukund S Chorghade (THINQ Pharma, USA) stated that, in recent years, a confluence of spectacular advances in chemistry, molecular biology, genomic and chemical technology and the cognate fields of spectroscopy, chromatography and crystallography have led to the discovery and development of numerous compounds and technologies. Multidisciplinary and multifunctional teams focusing on optimization have replaced the traditional specialized research groups. To 
progress from conception to commercialization, the entrepreneurial industry has reached out and established global strategic partnerships with numerous companies overseas. Process chemistry/route selection are important activities in the path of a drug from discovery to market. The synthetic routes employed in medicinal chemistry are usually low yielding and fraught with capricious reactions, tedious chromatography and problems in scale-up. He exemplified this by discussing the route-selection efforts on anti-asthma and antiphthalassemia drug candidates.

\section{Role of safety \& security in chemical sustainability}

Nancy B Jackson (Sandia National Laboratories, NM, USA) discussed 'The Role of Safety and Security in Chemical Sustainability.' She highlighted the importance of making chemical processes and industries more environmentally benign with the adoption of safe practices in the chemistry laboratory and the secure management of chemicals. She presented that the safe use of chemicals is necessary not only for the health and safety of scientists, but also in order to maintain community relationships and protect the environment. The best safety and security is achieved through a culture of safety and not by a rule-based compliance attitude.

\section{Lessons from DNA damage applied to siRNA}

Cynthia J Burrows (University of Utah, UT, USA) discussed the lessons from DNA damage applied to siRNA. She discussed that knockdown of specific genes of interest by siRNA is one very promising approach for drug design. A series of 8-oxo-2'deoxyguanosine and other purine analogs has been synthesized and incorporated into various positions of siRNA known to knockdown caspase-2. The results obtained from these experiments will be used for designing better siRNA with fewer off-target effects and better knockdown capability.

\section{Oncologic drug development: from target identification}

Tsann-Long Su (Institute of Biomedical Sciences, Academia, Taiwan), delivered his lecture on cancer, commenting that cancer is one of the top ten leading causes of death in the world and many strategies for treating cancer patients have been applied clinically. The advances in the treatment of malignant diseases have been limited owing to the failure to identify unique biochemical and/or biological properties that are able to clearly distinguish cancers from normal cell population.
Consequently, currently available anticancer agents lack tumor selectivity and possess a narrow therapeutic index. He highlighted that in recent years there have been marked advances in the understanding of tumor cell biology and oncogenes, leading to the identification of novel targets for designing new anticancer agents.

\section{Synthetic tools that modulate the} action of glycan-metabolizing enzymes Aloysius Siriwardena (Université de Picardie Jules Verne, Amiens, France) discussed the design and synthesis of chemical prototypes that modulate the action of oligosaccharidemetabolizing enzymes. He presented that natural products with inhibitory activity against glycosyl hydrolases $(\mathrm{GH})$ have been the primary inspiration for the design of analogs promising to display improved selectivity and potency for their target $\mathrm{GH}$.

Andrea Vasella (Zurich, Switzerland) presented on the synthesis of analogues where the nucleic acid backbone is replaced by elements directly linking nucleobases to each other and evaluating the pairing of such analogs to form defined structural elements. He highlighted that the ultimate goal is the design and synthesis of analogs of this type that possess useful properties against several diseases.

Sham Nikam (Nycomed, Germany) discussed the optimization of chemical leads in multitarget strategies. He highlighted the efforts of a discovery team to identify a preclinical lead compound, a multi-target agent. The complex pharmacology was a major hurdle but systematic SAR studies led to a lead compound that had significantly lower side-effect potential.

\section{ISCB award lectures}

The ISCB awarded Ajayan Vinu (National Institute for Materials Science, Namiki, Tsukuba, Japan) the ISCB award for excellence in chemical sciences. The 2010 ISCB young scientist in drug research award went to Diwan Singh Rawat (Delhi University, Delhi, India).

Ajayan Vinu discussed carbon and boron nitrides (BN) in his award lecture; highlighting these as very interesting materials that have attracted worldwide attention because the incorporation of nitrogen atoms into the carbon or boron nanostructure can enhance the mechanical, conducting, field emission and energy storage properties. However, only nonporous nitride materials have so far been reported. In his award lecture he presented the results on the preparation and characterization of nanoporous $\mathrm{CN}$, 
$\mathrm{BN}$ and BCN which were prepared by a novel nanohard templating technique. The discovery of novel nanoporous pure carbons such as carbon nanocoops, carbon nanocages and glucocarbons that exhibit extremely high surface area, large pore volume and uniform pore diameter was presented.

Diwan Singh Rawat discussed tetraoxane, a relatively less explored class of compounds that has been the subject of intense study due to its artemisinin-like antimalarial activity. In order to generate structural diversity in this class of compounds, tetraoxanes have been synthesized using less expensive benzaldehyes as starting materials. This work has resulted in many antimalarial agents that have shown promise as lead compounds.

Nancy B Jackson, president elect of the American Chemical Society, Colin J Suckling, Michael D Threadgill (UK), Mukund Chorghade (USA), Anamik Shah, VicePresident ISCB and PMS Chauhan, General Secretary of the ISCB all participated in the conference's farewell address. In the valedictory function all participants highlighted the importance of interaction between scientists from biological sciences, chemical sciences and pharmaceutical sciences. Most of the participants discussed how the process of drug research has become more difficult, risky and expensive due to very tight regulatory parameters. To make a breakthrough in this area, close interaction between scientists and technologists in chemistry and biology and other related areas is highly desired.

A number of renowned pharmaceutical companies, including Ranbaxy, Jubilant Chemsys Limited, ZydusCadila, Orion Pharma, Nicholas Piramal, GVK Biosciences Pvt. Ltd.,
Torrent Pharmaceuticals Ltd., Wockhardt Aurangabad and THINQ Pharma, participated in this conference.

\section{Looking ahead}

The next conference in the series will be entitled 'Interface of Chemistry and Biology in Drug Research.' It will be held at Saurashtra University, Rajkot (Gujrat, India).

\section{Conclusion}

The close interaction of scientists with varied interests in diverse fields of research is important. This conference has provided a common platform and opportunities for researchers in the areas of chemical and biological sciences and other related areas to interact with each other for mutual benefit. Apart from eminent academics, a number of professionals from the pharmaceutical and biotechnology industry also actively participated. The delegates, particularly young researchers, felt that multidisciplinary collaboration is very important in modern drug research. The conference also provided an opportunity for eminent and young Indian researchers to interact. This direct interaction will be a great help for future research activity as well as the establishment of new collaborative research programs.

\section{Financial \& competing interests disclosure}

The author has no relevant affiliations or financial involvement with any organization or entity with a financial interest in or financial conflict with the subject matter or materials discussed in the manuscript. This includes employment, consultancies, honoraria, stock ownership or options, expert testimony, grants or patents received or pending, or royalties.

No writing assistance was utilized in the production of this manuscript. 